\title{
The suppression of kindling with low-frequency brain stimulation: Statistical data with intertrial intervals variable
}

\author{
JOHN GAITO \\ York University, Downview, Ontario, Canada
}

The use of 1- or $3-\mathrm{Hz}$ brain stimulation has been effective in eliminating or preventing kindling behavior produced by $60-\mathrm{Hz}$ stimulation. Statistical data are presented for a number of experiments with an important contributor to this suppression effect, intertrial interval for stimulation. For experimental rats, each experiment involved the sandwiching of the kindling producing agent $(60-\mathrm{Hz}$ sine wave stimulation) between trials of the suppression-producing agent $(1-\mathrm{Hz}$ sine waves) (i.e., a sequence of 1-60-1). The intervals used were 1, 3, 24, 72, 120, and $336 \mathrm{~h}$; a control group received only $60-\mathrm{Hz}$ stimulation. The greatest degree of suppression occurred with $3 \mathrm{~h}$ between the $1-\mathrm{Hz}$ and $60-\mathrm{Hz}$ stimulations. One-hour intervals produced substantial suppression, whereas 24-h intervals effected little suppression and 72-, 120-, and 336-h groups were either unaffected or affected only minimally. Recovery from suppression following 15 or 16 days of nonstimulation appeared to be complete for the 1-h group; incomplete recovery was present with 3and 24-h intervals.

With periodic stimulation by $60-\mathrm{Hz}$ sine waves of an animal's specific brain sites (e.g., the amygdala), behavior changes from exploratory behavior (Stage 1), to automatic behaviors (Stage 2), and finally to clonic convulsions (Stage 3). In the process of studying this kindling event, we found that low frequency sine wave stimulation suppresses or prevents kindled behavior. Recently, we discussed the contribution of the duration of stimulation variable in the suppression process (Gaito, 1985). In general, as the period of stimulation increased from 15 to $600 \mathrm{sec}$, the suppression tendency deepened. In the case of shorter durations (up to $60 \mathrm{sec}$ ), the recovery process after a period of 15 or 16 days of nonstimulation appeared to be complete, or nearly so. With longer durations, incomplete recovery occurred.

The recovery from suppression following rest intervals suggested that the suppression event was a transient one. If a time-dependent suppression process is involved, one would expect that suppression would be reduced or eliminated as the intertrial interval is increased. In this paper we provide statistical analyses for another important contributor in the suppression process, the intertrial interval, which is defined as the interval between stimulation with the suppression agent $(1-\mathrm{Hz}$ sine waves) and the kindling agent $(60-\mathrm{Hz}$ sine waves).

\section{METHOD}

In each experiment, male Wistar rats (between 90 and 140 days of age) had nichrome bipolar electrodes placed unilaterally in the amygdala. The brain coordinates for electrode implantation were the same as in many experiments in our laboratory: $.5 \mathrm{~mm}$ posterior to bregma, $4.5 \mathrm{~mm}$ from midline, $8.5 \mathrm{~mm}$ from skull (bregma as reference point).

The author thanks Judith Emslie for calculating the means and standard deviations reported in this paper. The author's mailing address is: York University, Downsview, Ontario, Canada M3J 1P3.
Stimulation was not imposed until at least seven days after surgery. Then the rats were stimulated with $60-\mathrm{Hz}$ sine waves for $30 \mathrm{sec}$ during three trials on the first day. One hour intervened between trials. A Lafayette stimulator was used; the intensity was $36 \mu \mathrm{A}$ (root mean square, RMS; equivalent to $100 \mu \mathrm{A}$, peak to peak). On the first trial of the second day, the first effective threshold intensity $\left(\mathrm{ETI}_{1}\right)$ was determined. The $60-\mathrm{Hz}$ current was increased until a Stage 2 or 3 response was elicited. Then, $5 \mu \mathrm{A}$ was added to allow for day-to-day threshold fluctuations. Two further trials of stimulation at this intensity were provided to check on ETI $_{1}$ stability. The rats were separated into two groups by pairing most rats so as to maintain approximately equal mean ETI values for the two groups.

Then, one group of rats received stimulation with $1-\mathrm{Hz}$ sine waves for $120 \mathrm{sec}$ on Trials 1 and 3, 4 and 6,7 and 9, 10 and 12, and 13 and 15 at twice the $\mathrm{ETI}_{1}$ value. A $60-\mathrm{Hz}$ stimulation trial was provided on Trials 2, 5, 8, 11, and 14 for $30 \mathrm{sec}$ at $\mathrm{ETI}_{1}$ (Group 1, 1-60-1). This procedure involved a $60-\mathrm{Hz}$ stimulation trial sandwiched between $1-\mathrm{Hz}$ stimulation trials. In these experiments, intertrial intervals between 1$\mathrm{Hz}$ and $60-\mathrm{Hz}$ brain stimulation was the variable of concern. Groups of rats had 1-, 3-, 24-, 72-, 120-, and 336-h intervals between stimulation events. A control group had $60-\mathrm{Hz}$ stimulation on the same trials as the 1-60-1 group, but for the other trials each rat was placed in the apparatus without stimulation (Group 2, X-60-X). All 60-Hz stimulation was at $\mathrm{ETI}_{1}$ for $30 \mathrm{sec}$, a duration that has been used routinely in our research. Stimulation with $1-\mathrm{Hz}$ current was for 120 -sec duration at two times $\mathrm{ETI}_{1}$; this duration and intensity have been found to produce a drastic suppressive effect in previous experiments.

Following these 15 trials, rats from groups with 1-, 3-, and 24-h intervals had $\mathrm{ETI}_{2}$ determined over 6 trials during two days ( 3 trials/day). Then another block of 15 trials of stimulation occurred in which rats in each of these groups were treated in the same fashion as in the previous block of trials prior to the ETI ${ }_{2}$ determination. This alternation of ETI determinations and a block of trials was continued through the ETI determination. Following a rest interval of 15 or 16 days for these rats, ETI $_{5}$ was determined.

Because of the lengthy intertrial intervals of 120 and $336 \mathrm{~h}$, rats in these groups had only one set of 15 trials and ended the experiment with the $\mathrm{ETI}_{2}$ determination.

At the end of early experiments, histological analyses were performed on all rats. However, electrode tips were in the amygdala in almost all cases; also no gross lesions were detected at intensities of $200 \mu \mathrm{A}$ (RMS) and below, intensities that are used routinely in our experiments. The tissue around the electrode tips in rats stimulated with $1-\mathrm{Hz}$ or $3-\mathrm{Hz}$ 
and with $60-\mathrm{Hz}$ current was indistinguishable from that of rats stimulated with only $60-\mathrm{Hz}$ current. Thus, no histological analyses were conducted in the later experiments.

No intensities above $200 \mu \mathrm{A}$ were used for 1 - or $60-\mathrm{Hz}$ stimulation. Rats which showed Stage 1 behavior during an ETI determination with $60-\mathrm{Hz}$ stimulation were given a value of $208 \mu \mathrm{A}$. Thus, the upper limit for recorded ETI with any rat was $208 \mu \mathrm{A}$.

\section{RESULTS AND DISCUSSION}

The mean ETI values are shown in Table 1. Table 2 presents the standard deviations. The ratio of a specific ETI value to that for $\mathrm{ETI}_{1}$ indicates the relative change. These values are present in Table 1 also.

The data in Table 2 for standard deviations do not show any systematic trends. However, based on statistics presented in Table 1, there are a number of points worth mentioning.

Suppression Effect. (1) The control group indicates a slight and gradual decline in mean ETI values over the four ETI determinations. This decline is typical. (2) The 1 -h group shows the typical great increase in mean ETI values over the determinations. This interval is the one which had been used for many experiments in our laboratory. (3) The 3-h interval effects the greatest suppression result. The ratio at $\mathrm{ETI}_{4}$ (after the last phase of 1-Hz stimulation) reaches 5.15, a tremendous increase in mean threshold value. (4) The $24-$ and $72-\mathrm{h}$ periods provide a slight suppression result. (5) The 120 - and 336 -h periods

Table 1

Mean ETI Values and ETI/ETI 1 Ratios for Various Intertrial Intervals between 1-Hz and $60-\mathrm{Hz}$ Brain Stimulation

\begin{tabular}{lcccccc}
\hline \multirow{2}{*}{$\begin{array}{l}\text { Intertrial } \\
\text { Interval }(\mathrm{h})\end{array}$} & $\mathrm{n}$ & 1 & 2 & 3 & 4 & 5 \\
\hline & \multicolumn{6}{c}{ ETI Determinations } \\
\hline Control & 7 & 41.4 & 33.6 & 30.0 & 30.7 & 37.1 \\
& & 1.00 & .812 & .725 & .742 & .896 \\
1 & 6 & 77.2 & 141.0 & 195.7 & 198.3 & 67.5 \\
& & 1.00 & 1.83 & 2.53 & 2.57 & .874 \\
3 & 8 & 40.0 & 90.0 & 172.6 & 206.0 & 86.3 \\
& & 1.00 & 2.25 & 4.32 & 5.15 & 2.16 \\
24 & 9 & 78.3 & 82.2 & 93.3 & 117.8 & 100.6 \\
& & 1.00 & 1.05 & 1.19 & 1.50 & 1.28 \\
72 & 17 & 108.5 & 100.0 & 87.9 & 79.7 & \\
& & 1.00 & .922 & .810 & .735 & \\
120 & 4 & 77.5 & 59.5 & & & \\
336 & 7 & 95.0 & 83.6 & & & \\
& & 1.00 & .880 & & & \\
\hline
\end{tabular}

Table 2

Standard Deviations for Various Intertrial Intervals between 1-Hz and 60-Hz Brain Stimulation

\begin{tabular}{lccccc}
\hline & \multicolumn{5}{c}{ ETI Determinations } \\
\cline { 2 - 6 } Intertrial & 1 & 2 & 3 & \multicolumn{1}{c}{4} & 5 \\
\hline Control & 20.8 & 16.8 & 12.6 & 12.7 & 14.4 \\
1 & 45.9 & 62.8 & 22.7 & 23.7 & 41.8 \\
3 & 14.9 & 53.7 & 44.5 & 3.7 & 22.6 \\
24 & 55.5 & 40.9 & 31.6 & 36.3 & 56.5 \\
72 & 63.7 & 50.6 & 44.1 & 41.2 & \\
120 & 51.9 & 30.1 & & & \\
336 & 48.7 & 43.7 & & & \\
\hline
\end{tabular}

Table 3

ETI/ETI Ratio Comparisons

\begin{tabular}{lccc}
\hline Intertrial & \multicolumn{3}{c}{ Ratios } \\
\cline { 2 - 4 } Intervals (h) & $2-1^{*}$ & $4-1^{* *}$ & $5-1^{* * *}$ \\
\hline Control & -.188 & -.258 & -.104 \\
1 & .83 & 1.57 & -.126 \\
3 & 1.25 & 4.15 & 1.16 \\
24 & .05 & .50 & .28 \\
72 & .078 & .265 & \\
120 & -.232 & & \\
336 & -.120 & & \\
\hline
\end{tabular}

${ }^{*} E T I_{2} / E T I_{1}$ minus $1.00{ }^{* *} E T I_{4} / E T I_{1}$ minus $1.00 . \quad * * * E T I_{5} / E T I_{1}$ minus 1.00 .

Table 4

Summary Sheet for Stimulation Effects Relative to to Intertrial Intervals at $120 \mathrm{Sec}$ Duration

\begin{tabular}{lll}
\hline Interval (h) & \multicolumn{1}{c}{ Suppression } & \multicolumn{1}{c}{ Recovery } \\
\hline Control & not relevant & not relevant \\
1 & great & complete \\
3 & greatest & not complete \\
24 & little effect & nearly complete \\
72 & little effect & \\
120 & no apparent effect & \\
336 & no apparent effect & \\
\hline
\end{tabular}

indicate a slight decline, suggesting no (or little) suppression occurring.

Recovery from suppression. A rest period of 15 or 16 days occurred between the fourth and fifth threshold determinations. Thus, $\mathrm{ETI}_{5} / \mathrm{ETI}_{1}$ provides one index of recovery from the suppression process. Table 1 shows the recovery events as follows: (1) Recovery appears to be complete at the $\mathrm{ETI}_{5}$ determination for the 1-h group. (2) Recovery is lowest for the 3-h interval group (possibly because the suppression process is greater). (3) Recovery is partial at 3- and 24-h intervals. Recovery indications are not available for the 72-, 120-, and 336-h groups, because ETI $_{5}$ values were not obtained.

The above effects are shown clearly in Table 3, which provides comparisons of the ratio relative to specific ETI determinations for the various intertrial intervals, and are summarized in Table 4 . The $\mathrm{ETI}_{2} / \mathrm{ETI}_{1}$ ratio minus the $\mathrm{ETI} / \mathrm{ETI}_{1}$ ratio $(1.00)$ indicates the magnitude of the short term suppression tendency, whereas the $4: 1$ comparison shows a longer term suppression effect. These two measures are suppression indices; positive values indicate suppression, with increasing degree related to increasing magnitude. The 5:1 comparison indicates the recovery from suppression, a recovery index. Values around zero, or below, indicate lack of residual suppression, that is, complete (or near complete) recovery; positive values indicate less than complete recovery, with less recovery for increasing values.

\section{REFERENCE}

GaITo, J. (1985). The suppression of kindling with low frequency brain stimulation: Statistical data with duration variable. Bulletin of the Psychonomic Society, 23, 332-334.

(Manuscript received for publication March 25, 1985.) 